\title{
Cycloconvertisseurs mixtes thyristors/GTO à haut facteur de puissance
}

\author{
Frédéric Richardeau $\left(^{*}\right)$ et Yvon Chéron \\ Laboratoire d'Électrotechnique et d'Électronque Industrielle $\left({ }^{* *}\right)$, INP de Toulouse, \\ ENSEEIHT, 2 rue Charles Camichel, 31071 Toulouse Cedex, France
}

(Reçu le 10 juillet 1996, révısé le 29 octobre 1996, accepté le 18 décembre 1996)

\author{
PACS.07.50 Yd - Other electrical and electronic components \\ PACS.84.30 Jc - Power electronics \\ PACS.84.60.Bk - Performance characteristıcs of energy conversion systems
}

\begin{abstract}
Résumé. - Cet article présente une technique de suppression de la puissance réactive au sein même de convertisseurs à contrôle de phase tels que les commutateurs de courant et les cycloconvertisseurs. Le concept repose sur l'association de cellules d'interrupteurs à mécanismes de commutation duaux au moyen de thyristors (commutation naturelle au zéro du courant) et de GTO symétriques (commutation naturelle au zéro de la tension aux bornes). Les cellules à thyristors travaillent avec un angle de retard à l'amorçage et consomment de la puissance réactive. À l'opposé, les cellules à GTO utilısent un angle d'avance à l'amorçage et produsent de la puissance réactive. La puissance réactive moyenne résultante est nulle et le facteur de puissance se réduit au facteur de forme du courant de ligne. L'extension aux montages cycloconvertısseurs conduit à trois structures de base. Parmi celles-ci, deux présentent l'avantage d'être naturellement réversibles en courant. Ces deux structures utilisent soit une association de ponts complets d'indice de pulsation 6 , mixtes entre eux, soit une association de ponts mixtes d'indice de pulsation 3. La première association produit un facteur de puissance élevé aux dépends d'une ondulation basse fréquence de puissance réactive. La seconde association ne possède pas cet inconvénient avec cependant un facteur de puissance moindre La caractérisation et la comparaison de ces structures ont été validées par un prototype de laboratoire triphasé-triphasé à un niveau de puissance de $20 \mathrm{kVA}$.
\end{abstract}

\begin{abstract}
This paper deals with a reactive power suppression technique inside phasecontrolled structures such as rectifier bridges and cycloconverters. The concept is based on an association between dual-switching cells by means of thyristors (natural zero current switching) and reverse blocking abilties GTOs (natural zero voltage switching). Thyristor cells work with a lagging phase shifting control and draw a positive reactive power. On the contrary, GTO cells use a leading phase shifting control and draw a negative reactive power. The total average reactive power in the power system is zero and the power factor is only given by the line current form factor. The application of this concept to cycloconverters gives three basic structures. Two of them have a natural current reversibility. These two structures use, either a mixed full bridges association with a pulse number of 6 , or a mixed bridges association with a pulse number of 3 . The first one gives a high power factor but draws a low frequency fluctuating reactive power. The second one yields no reactive power but with a less power factor. The study and the comparison of these structure have been carried out on a $20 \mathrm{kVA}$ three-phase laboratory prototype.
\end{abstract}

( ${ }^{*}$ Auteur auquel doit être adressée la correspondance (e-mail : richard@leei.enseeıht.fr)

$\left({ }^{* *}\right)$ UPRES-A au CNRS $N^{\circ} 5004$

(C) Les Éditions de Physique 1997 


\section{Introduction}

Dans le domaine de la conversion statique de forte puissance $(P>500 \mathrm{~kW})$, les montages à thyristors en contrôle de phase sont reconnus pour leur grande fiabilité, leur facilité de mise en œuvre et leur rendement élevé. Cependant, ils souffrent de graves défauts :

- le fonctionnement des interrupteurs à la fréquence de la source d'alimentation produit des courants harmoniques de rang bas, particulièrement difficiles à filtrer ;

- le réglage du transfert de puissance s'effectue par le retard de phase entre les courants de ligne et leurs tensions respectives [1] provoquant alors la circulation d'une puissance réactıve.

Pour les convertisseurs directs utilısés en forte puissance tels que les commutateurs de courant et les cycloconvertisseurs [2], ces deux nuisances conduisent à dégrader le rendement électrique ou facteur de puissance du montage.

Bien que des solutions existent pour atténuer ces perturbations (augmentation de l'indice de pulsation, décalage des ordres de commande) [1], cet article propose une nouvelle approche du problème reposant sur une association d'interrupteurs à mécanismes de commutation duaux au sein du convertisseur. Cette action porte uniquement sur la suppression de la pussance réactive tout en préservant le fonctionnement en contrôle de phase et donc à pertes réduites sur les interrupteurs.

Après la présentation du concept, l'article s'oriente vers l'étude comparative et l'expérimentation de structures génériques de cycloconvertisseurs à puissance réactive nulle

\section{Ponts redresseurs mixtes ZCS-ZVS}

2.1. INTERRUPteurs À COMMUTATIONS DUALES. - Les caractéristiques de commutation du thyristor sont rappelées sur la figure 1a. L'amorçage est commandé tandis que le blocage s'effectue spontanément au passage par zéro du courant dans l'interrupteur (mode ZCS : Zero Current Switching). Sur cette base, il est possible de définir un interrupteur ayant des caractéristiques dynamiques duales représentées sur la figure $1 \mathrm{~b}:$ un amorçage spontané sous tension nulle de l'interrupteur et un blocage commandé (mode ZVS : Zero Voltage Switching). Cet interrupteur ayant les mêmes caractéristiques statiques que le thyristor, il peut donc être utilisé dans toutes les structures de conversion directe raccordées au réseau Un tel interrupteur sera par la suite désigné par TDD (Thyristor-Diode-Dual).

La synthèse du TDD peut s'opérer au moyen d'une fonction transistor (bipolaire, IGBT, MCT) associée avec une diode en série ou bien plus naturellement avec un GTO symétrique en tension muni d'une logique d'autopilotage similaire à celle du thyristor-dual [4]. Contrairement au mode ZCS, la nature inductive des sources d'alimentation industrielles est fondamentalement incompatible avec le mode ZVS et interdit toute interruption du courant (commande au blocage). Un découplage passif ou un dispositif d'écrêtage placé en entrée du montage est donc indispensable en complément du convertisseur.

L'utilisation d'un filtre passif de découplage de type LC pose deux problèmes fondamentaux. Le premier problème repose sur le choix de la fréquence de résonance parallèle du filtre. Pour que le filtre soit efficace, cette fréquence doit être comprise entre la fréquence du fondamental et la fréquence du premier harmonique caractéristique de courant. En présence de courants harmoniques de rang bas, l'efficacité du filtrage est très limitée et on obtient un filtre très volumineux et donc onéreux Le deuxième problème tient à la proximité des harmoniques de courant. Lors d'une variation du point de fonctionnement du convertisseur, les harmoniques de courant de ligne sont modulés par la fréquence du régime transitoire. Ces harmoniques peuvent donc être transitoirement amplifiés par le filtre de découplage. 

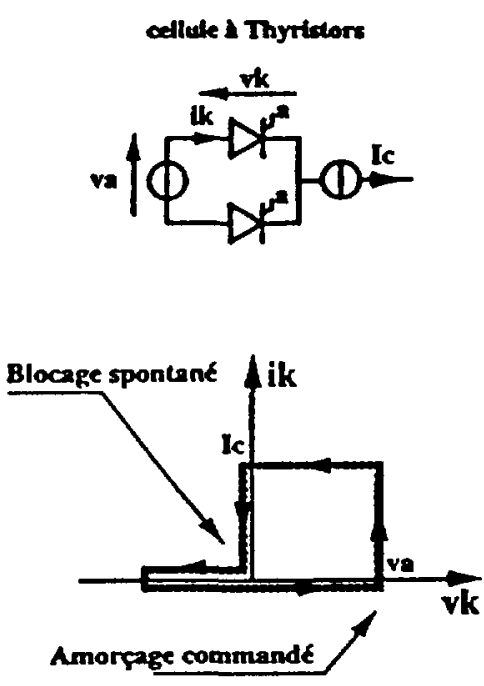

a)

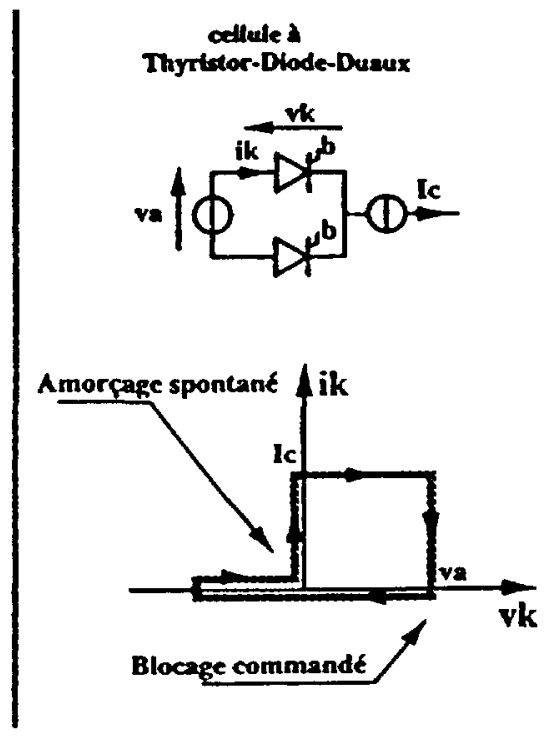

b)

Fig 1. - a) Cellule de commutation à thyristors (mode ZCS). b) Cellule de commutation à TDD (mode ZVS).

[a) Thyrıstor switchıng cell (ZCS mode) b) Dual thyristor-diode switching cell (ZVS mode).]

Pour contourner ces deux problèmes, on peut utıliser un écrêteur de tension placé entrephases à l'entrée du montage. Ce dispositif réalise un découplage du réseau uniquement lors de l'ouverture d'un des TDD et garantit la maîtrise de la tension aux bornes des interrupteurs quelles que sorent les conditions de fonctionnement du montage. L'écrêteur est constitué d'un pont de diodes suivi d'un condensateur dont la tension aux bornes est régulée, soit par un hacheur dissipatif, soit par un onduleur de récupération [6].

2.2. Ponts redresseurs À TDD et thyristors-TDD. - Puisque les cellules de commutation regroupent des interrupteurs de même type (thyristors ou TDD), deux structures de ponts redresseurs en contrôle de phase peuvent être définies :

- le pont complet à TDD (Fig. 2a),

- le pont mixte à thyristors et TDD (Fig. 2b).

La première structure présente la particularité de fournir de la puissance réactive à la source d'alimentation $(Q<0)$. Par conséquent, une association avec un pont complet à thyristors permet de compenser globalement le réactif avec un indice de pulsation $p=6$, moyennant au total 12 interrupteurs. La seconde structure possède des commutations de courant alternativement de type ZCS et ZVS à l'échelle de la période d'alimentation. Ainsi, en désignant respectivement par $\alpha_{\text {on }}$ et $\alpha_{\text {off }}$ l'angle de contrôle des thyristors et des TDD, la suppression de la puissance réactive est obtenue avec seulement 6 interrupteurs à condition de vérifier :

$$
\alpha_{\mathrm{off}}=\pi-\alpha_{\mathrm{on}}
$$




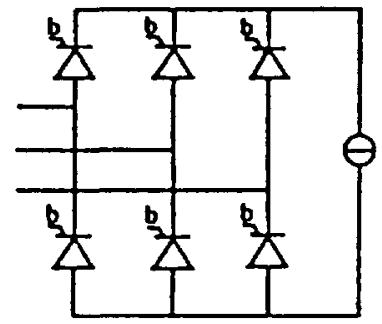

a)

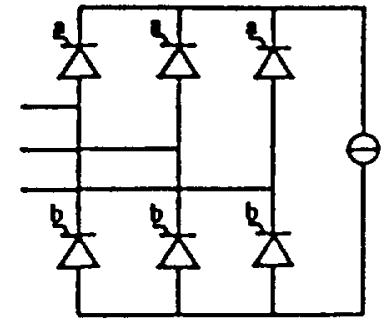

b)

Fig. 2. - a) Pont redresseur complet à TDD. b) Pont redresseur mixte à thyristors et TDD [a) Dual thyristor-diode full bridge. b) Mixed thyrıstor/dual thyristor-diode bridge.]

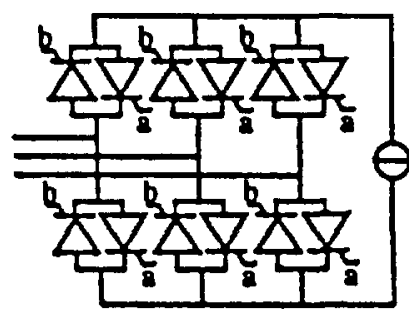

a)

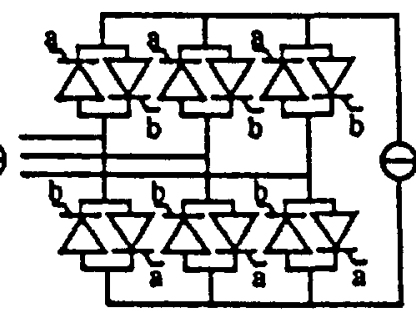

b1)

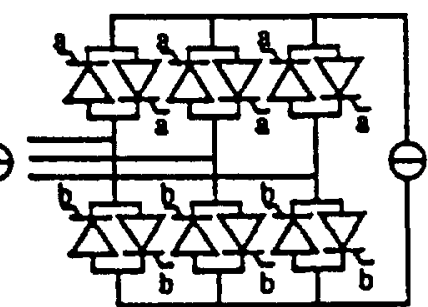

b2)

Fig. 3. - a) Cycloconvertisseur en ponts complets. b) Cycloconvertisseurs en ponts mixtes (deux versions).

[a) Full bridges cycloconverter. b) Mixed bridges cycloconverters.]

Malheureusement dans le second cas, cette mixité de commutation s'accompagne d'un indice de pulsation réduit à $p=3$ avec l'apparition de rangs pairs sur les courants de ligne [5].

En fait, à puissance donnée, bien que dans la première structure il faille deux fois plus d'interrupteurs, ceux-ci sont dimensionnés avec un calibre en tension ou en courant moitié de ceux des interrupteurs de la seconde structure. Cela signifie que les deux structures ont une puissance "silicium" totale identique.

\section{Cycloconvertisseurs mixtes thyristors-TDD}

3.1. Définition des StRuctures. - Sur la base des structures de ponts redresseurs de la figure 2, trois cycloconvertisseurs mixtes à thyristors et TDD peuvent être recensés (Fig. 3). La caractéristique commune aux structures précédentes est la suppression de la puissance réactive moyenne. Toutefois, celles-ci possèdent des propriétés spécifiques, tant sur le plan des composantes harmoniques, du facteur de puissance et des facilités de mise en œuvre qui vont être exposées dans les paragraphes suivants.

L'étude repose sur un environnement idéalisé : la source de tension est parfaite (impédance nulle) de valeur efficace $V$ et la charge est identifiée à une source de courant $i_{0}$ de valeur efficace $I_{0}$. En outre, on désigne respectivement par $\omega_{1}$ et $\omega_{0}$, la pulsation d'entrée et de sortie du cycloconvertisseur et par $\mathcal{R}$ et $\cos \varphi_{0}$ le taux de modulation et le facteur de puissance de la charge. 

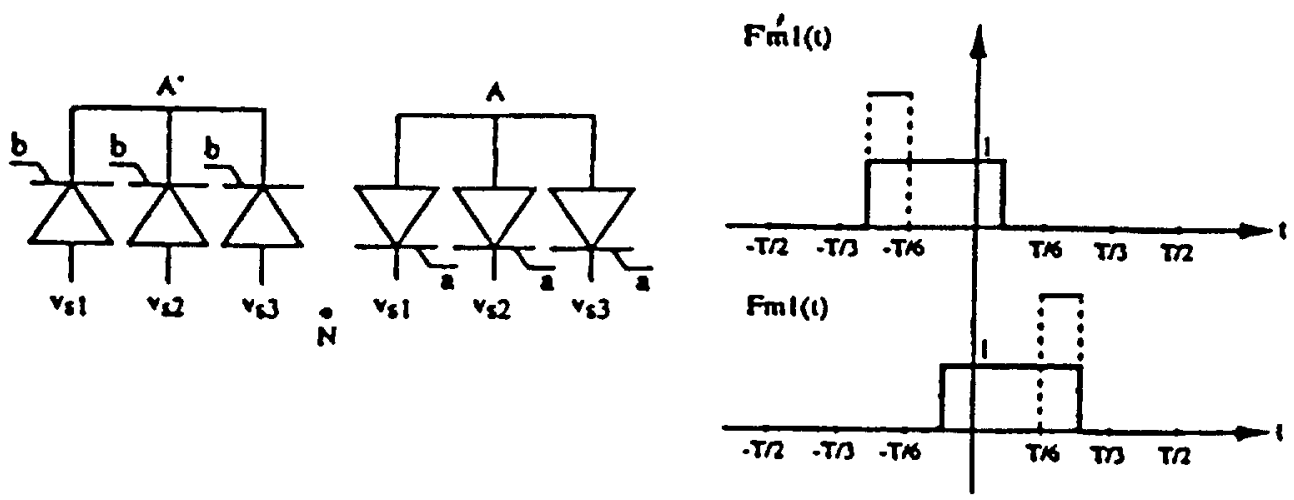

Fig. 4. - Fonctions de conduction des cellules ZCS $\left(F_{\mathrm{m} 1}\right)$ et ZVS $\left(F_{\mathrm{m}^{\prime} 1}\right)$. [Operating functions for ZCS $\left(F_{\mathrm{m} 1}\right)$ and ZVS $\left(F_{\mathrm{m}^{\prime} 1}\right)$ cells ]

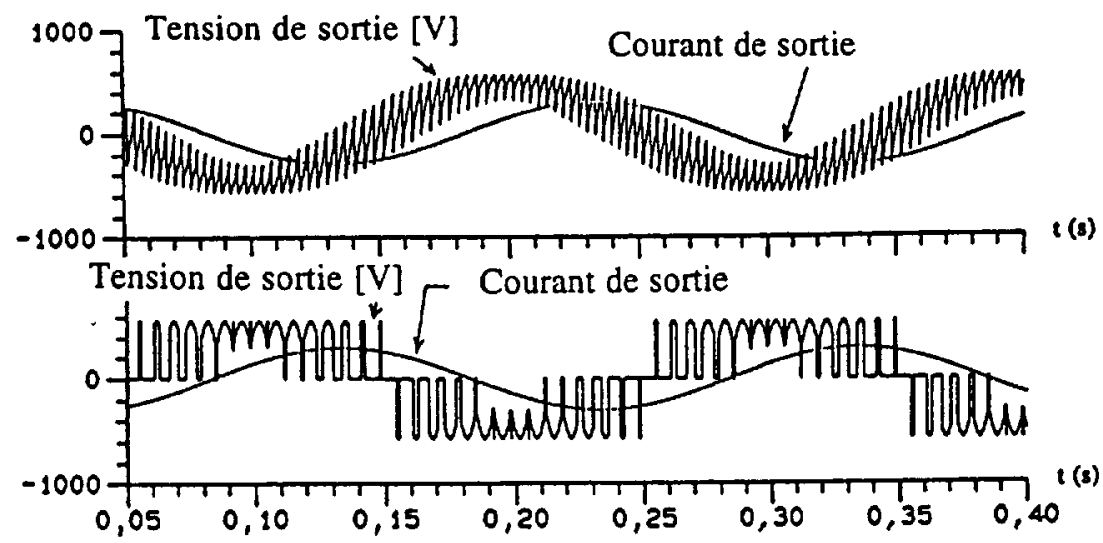

Paramètres : $f_{0}=5 \mathrm{~Hz}, \mathcal{R}=0,9, \varphi_{0}=60^{\circ}$.

Fig. 5. - a) Montage en ponts complets $(p=6)$. b) Montage en ponts mixtes $(p=3)$.

[a) Full bridges cycloconverter simulation results b) Mixed bridges cycloconverter simulation results.]

Les structures (a) et (b1) de la figure 3 sont constituées par une mise en antiparallèle de cellules à thyristors et de cellules à TDD. La conduction de chacun des interrupteurs de chaque cellule peut être définie par leur fonction de conduction ("0" ou "1") donnée sur la figure 4. La tension en valeur instantanée délivrée par chaque cellule est donnée par :

$$
\begin{gathered}
v_{\mathrm{AN}}^{\prime}=F_{\mathrm{m}^{\prime} 1} v_{\mathrm{s} 1}+F_{\mathrm{m}^{\prime} 2} v_{\mathrm{s} 2}+F_{\mathrm{m}^{\prime} 3} v_{\mathrm{s} 3}, \\
v_{\mathrm{AN}}=F_{\mathrm{m} 1} v_{\mathrm{s} 1}+F_{\mathrm{m} 2} v_{\mathrm{s} 2}+F_{\mathrm{m} 3} v_{\mathrm{s} 3} .
\end{gathered}
$$

Avec $F_{\mathbf{m}^{\prime} 1}$ :

$$
\frac{1}{3}-\frac{\sqrt{3}}{\pi}\left[\cos \left(\omega_{\mathrm{i}} t-\alpha_{\mathrm{off}}\right) \pm \sum_{h=1}^{\infty} \frac{1}{3 h \pm 1} \cos \left((3 h \pm 1)\left(\omega_{1}-\alpha_{\mathrm{off}}\right)\right)\right]
$$




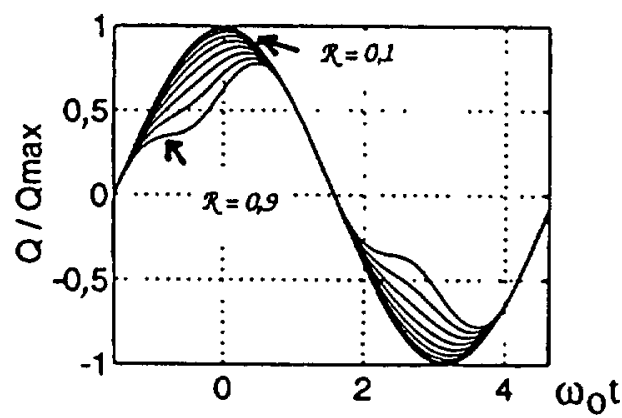

Structure monophasée

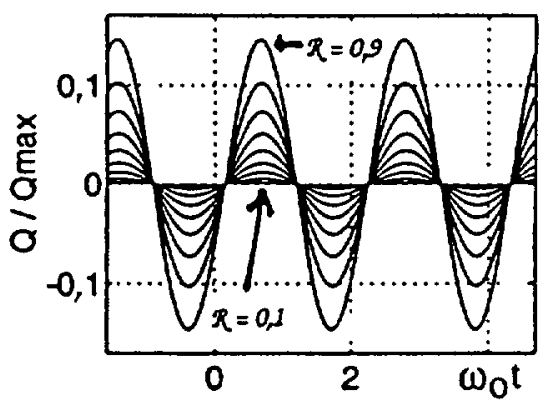

Structure triphasée

Fig. 6 - Puissance réactive "Instantanée" pour $\varphi_{0}=30^{\circ}$.

[Instantaneous reactive power for $\varphi_{0}=30^{\circ}$.]

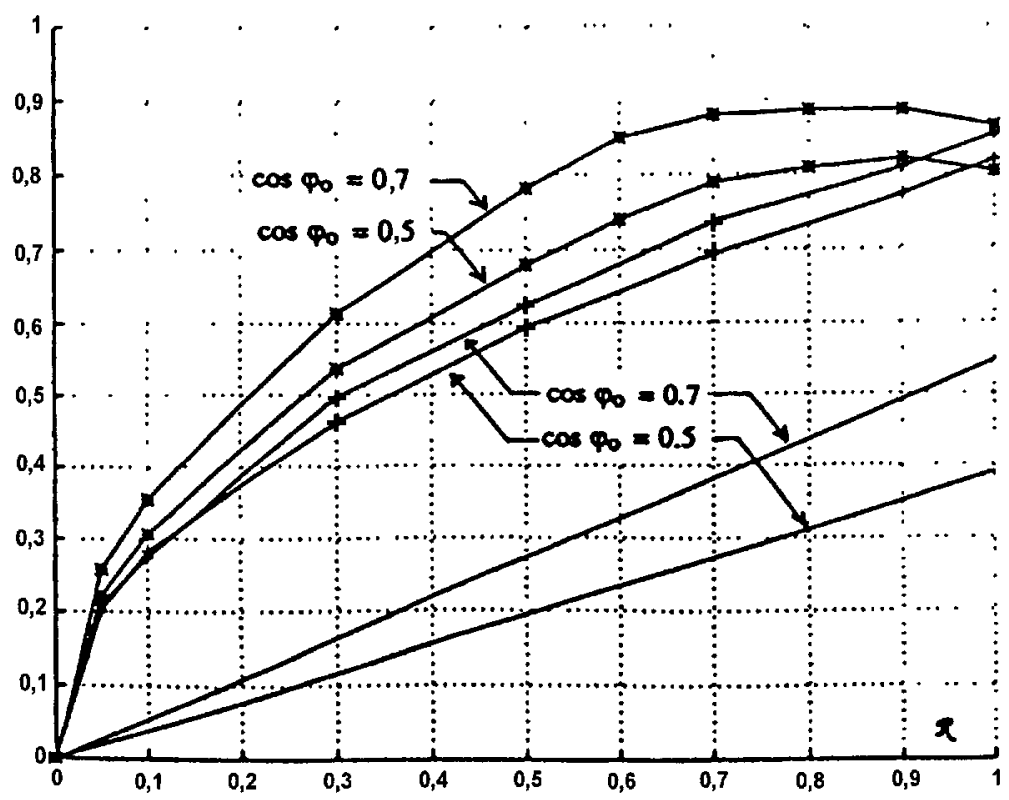

Fig. 7. - Comparaison du facteur de pussance, $(-)$ classique, $\left({ }^{*}\right)$ ponts complets, $(+)$ ponts mixtes [Power factor comparison, (-) classical, $\left(^{*}\right)$ full bridges, $(+)$ mixed bridges ]

et $F_{\mathrm{m} 1}$ :

$$
\frac{1}{3}-\frac{\sqrt{3}}{\pi}\left[\cos \left(\omega_{\mathrm{i}} t-\alpha_{\text {on }}\right) \pm \sum_{h=1}^{\infty} \frac{1}{3 h \pm 1} \cos \left((3 h \pm 1)\left(\omega_{1}-\alpha_{\text {on }}\right)\right)\right] .
$$

Les autres fonctions de conductions étant déphasées de $\pm 2 \pi / 3$ par rapport aux fonctions $F_{\mathrm{m}^{\prime} 1}$ et $F_{\mathrm{m} 1}$. À partir de (2) et (3), il est clair que l'identité des angles $\alpha_{\text {on }}$ et $\alpha_{\text {off }}$ permet de produire des tensions $v_{\mathrm{AN}}^{\prime}$ et $v_{\mathrm{AN}}$ identiques. Une mise en antiparallèle et une commande simultanée des thyristors et TDD est donc possible sans l'usage de procédé particulier. Les structures 


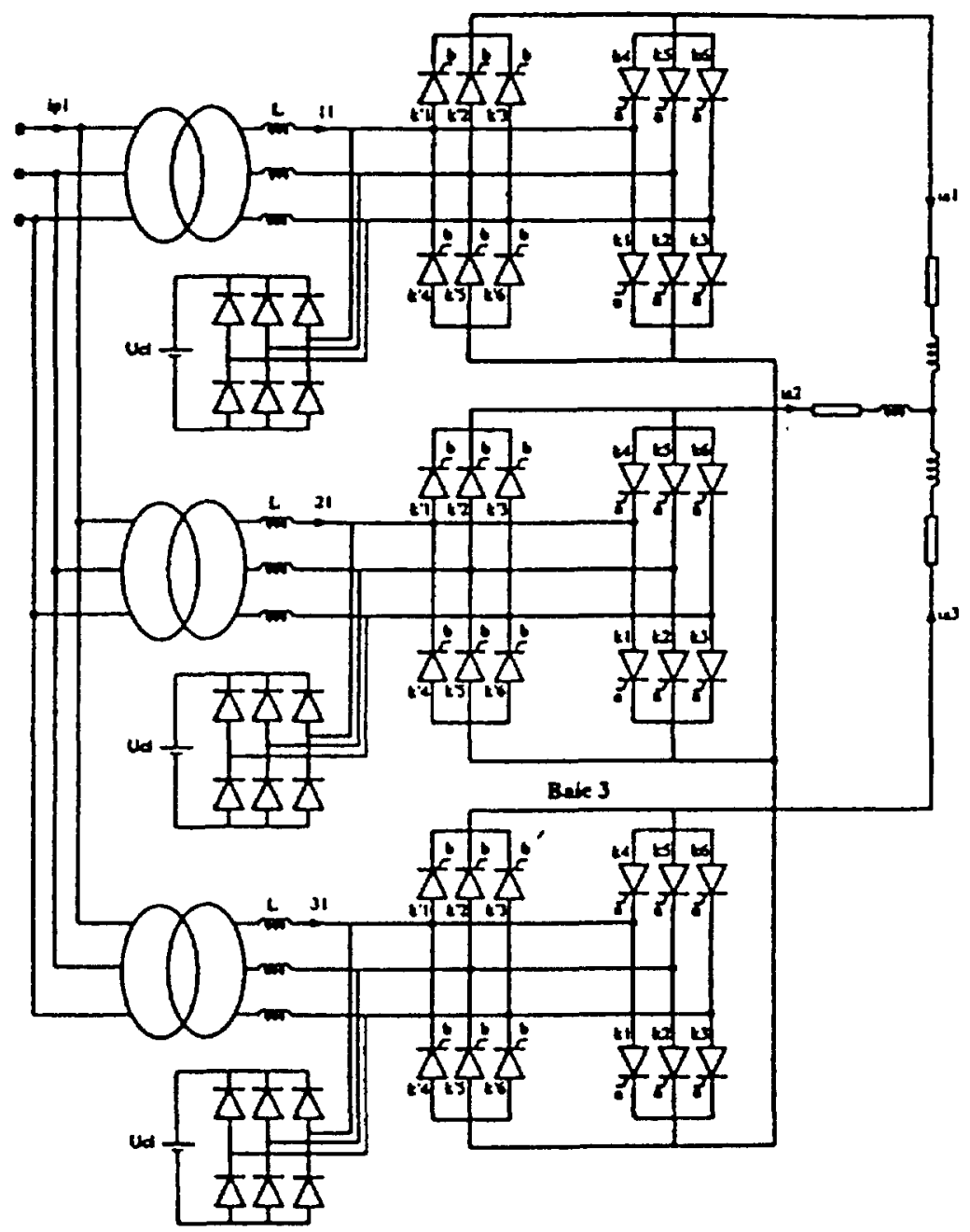

Fig. 8 - Prototype de cycloconvertısseur mixte ZCS-ZVS.

[ZCS-ZVS mixed mode cycloconverter prototype.]

(3a) et (3b1) ainsi définies sont donc naturellement réversibles en courant. Par contre, pour la structure (3b2), les cellules en antiparallèle étant de même type, une commande sélective par temps mort est nécessaire. La figure 5 reproduit la forme d'onde de tension délivrée par la structure en ponts complets (3a) et la structure en ponts mixtes (3b1).

3.2. STRUCTURE EN PONTS COMPLETS. - La puissance réactive produite par la structure est alternativement positive et négative selon le signe du courant $i_{0}$. Dans l'hypothèse où la fréquence de modulation $f_{0}$ est très faible par rapport à la fréquence d'alimentation, la puissance réactive peut être considérée comme lentement variable et a pour expression :

$$
Q(t)=Q_{\max } \cos \left(\omega_{0} t-\varphi_{0}\right) \sqrt{1-\frac{\mathcal{R}^{2}}{2}\left(1+\cos 2 \omega_{0} t\right)} .
$$


Avec $: Q_{\max }=\frac{6 \sqrt{3}}{\pi} V I_{0}$, dont le développement donne .

$$
Q(t)=\sum_{h=1}^{\infty} Q_{2 h-1} \cos \left((2 h-1) \omega_{0} t+\phi_{2 h-1}\right)
$$

L'extension au montage à sortie triphasée équilibrée revient en la superposition de trois formes d'ondes déphasées chacune de $\pm 2 \pi / 3$. Puisque seules les composantes de rang multiples de 3 donnent une somme non nulle, il vient :

$$
Q_{\text {total }}(t)=\sum_{h=1}^{\infty} Q_{3 h} \cos \left[3(2 h-1) \omega_{0} t+\phi_{3 h}\right] .
$$

La figure 6 reproduit les formes d'ondes de puissance réactive en version monophasée et triphasée. En version triphasée, ces résultats révèlent la présence d'une ondulation de réactif d'autant plus importante que le taux de modulation (et le niveau de puissance) est élevé.

3.3. Structure en ponts Mixtes. - En considérant que le courant de charge $i_{0}$ évolue très lentement à l'échelle de la période des tensions d'alimentation, le fonctionnement du cycloconvertisseur en ponts mixtes peut être assimilé, à tout instant, au fonctionnement d'un pont redresseur mixte. Dès lors, et contraırement à la structure en ponts complets, aucune puissance réactive n'est alors produite à tout instant.

\section{Facteur de puissance}

Le facteur de puissance produit par le cycloconvertisseur triphasé en ponts complets et le cycloconvertisseur en ponts mixtes a été déterminé par simulations numériques en opérant un balayage des paramètres $\mathcal{R}$ et $\varphi_{0}$. Les courbes sont données sur la figure 7 où se trouve également le facteur de puissance d'un montage classique.

La justification des courbes précédentes repose sur la remarque suivante : puisque les cycloconvertisseurs à mécanismes de commutation duaux ne mettent en jeu aucune puissance réactive moyenne, le facteur de puissance de ces structures se réduit au facteur de forme du courant de ligne. Ce facteur dépend de la topologie et de l'indice de pulsation du montage. Il est d'autant meilleur que l'indice de pulsation est élevé. Il est donc légitime d'obtenir un facteur de puissance meilleur pour la structure en ponts complets $(p=6)$ par rapport à la structure en ponts mixtes $(p=3)$. La structure en ponts complets présente un intérêt supplémentaire qui est son fonctionnement à facteur de puissance maximum constant sur une plage de fonctionnement significative : $\cos \varphi_{0} \approx 0,9$ pour $0,7<\mathcal{R}<0,9$. Cette propriété est très intéressante dans le cadre d'un entraînement à vitesse variable de moteur de forte puissance.

En conclusion, le tableau I récapitule les principales caractéristiques des structures étudiées.

\section{Résultats expérimentaux}

Les résultats expérimentaux sont issus d'un prototype de cycloconvertisseur triphasé-triphasé de $20 \mathrm{kVA}$ à structure modulaire (Fig. 8). La synthèse du mode TDD a été réalisée par des thyristors GTO symétriques en tension.

- alimentation $50 \mathrm{kVA}, U_{\mathrm{cc}}=3,5 \%$, 
Tableau I. - Récaptulatıf des propriétés des cycloconvertısseurs à mécanısmes de commutation duaux.

[Global characteristics on dual-switching cycloconverters.]

\begin{tabular}{|c|c|c|}
\hline Cycloconvertisseurs & Ponts complets & Ponts mixtes \\
\hline Indice de pulsation & 6 & 3 \\
\hline Ondulation de puissance réactive & oui (fonction de $\mathcal{R}$ et $\varphi_{0}$ ) & non \\
\hline Facteur de puissance & $\begin{array}{c}0,9 \text { max et quasi-constant } \\
\text { pour } 0,7<\mathcal{R}<0,9\end{array}$ & 0,85 max \\
\hline
\end{tabular}

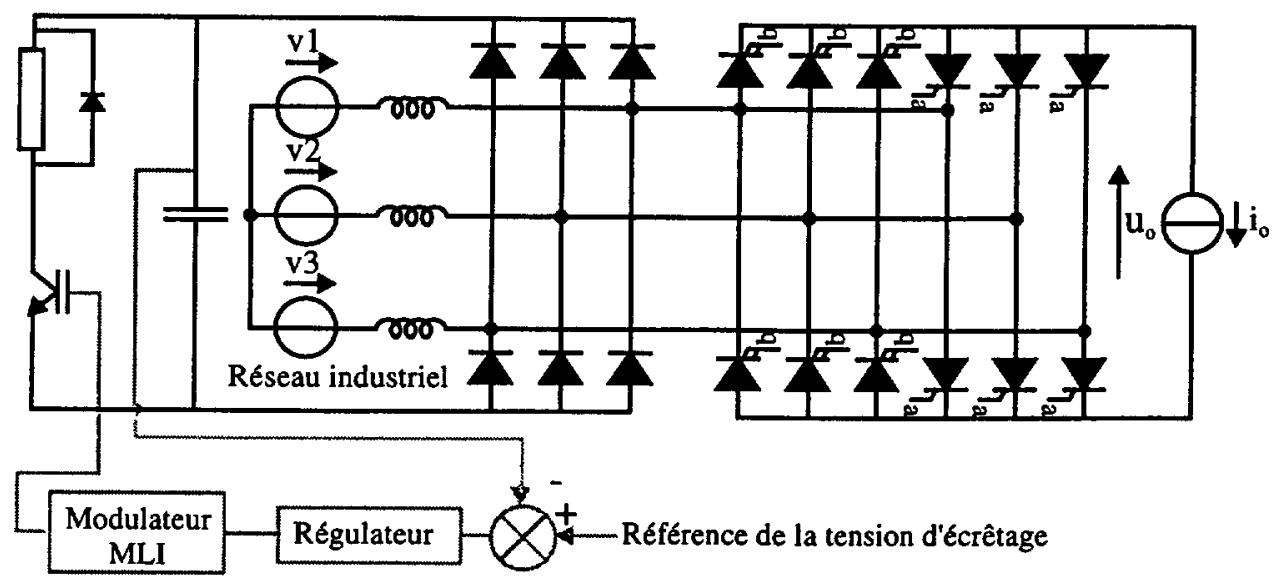

Fig. 9. - Écrêteur de tension associé à sa régulation.

[Clamping voltage device with this voltage loop.]

- transformateurs d'isolement $17 \mathrm{kVA}, U_{\mathrm{cc}}=3,2 \%$,

- GTO NIEC $1200 \mathrm{~V}-350 \mathrm{~A}$,

- thyristors NIEC $1200 \mathrm{~V}-200 \mathrm{~A}$.

Les écrêteurs de tension ont été réalısés au moyen de ponts de diodes raccordés à un condensateur aux bornes duquel la tension est régulée par un hacheur dissipatif. La tension est régulée à $750 V_{\max }$ aux bornes des interrupteurs. Les trois cycloconvertisseurs étant isolés, il est nécessaire de disposer un écrêteur par cycloconvertisseur. La figure 9 donne le schéma d'un écrêteur.

Les figures 10 et 11 représentent le fonctionnement en ponts complets en version triphasée équilibrée à un niveau de puissance de $20 \mathrm{kVA}$. La mesure du facteur de puissance a donné 0,86 contre 0,38 pour le montage classique tout thyristors. On peut noter la totale réversibilité en courant du montage. Les figures 12 et 13 représentent le fonctionnement en ponts mixtes.

Afin de cumuler les avantages des deux structures précédentes sans leurs inconvénients (réactif instantané nul, indice de pulsation de 6), la figure 12 propose une mise en série de cycloconvertisseurs en ponts complets. La permutation des ponts entre les deux cycloconvertisseurs permet de réaliser une circulation interne de la puissance réactive totalement invisible au niveau du réseau d'alimentation. 


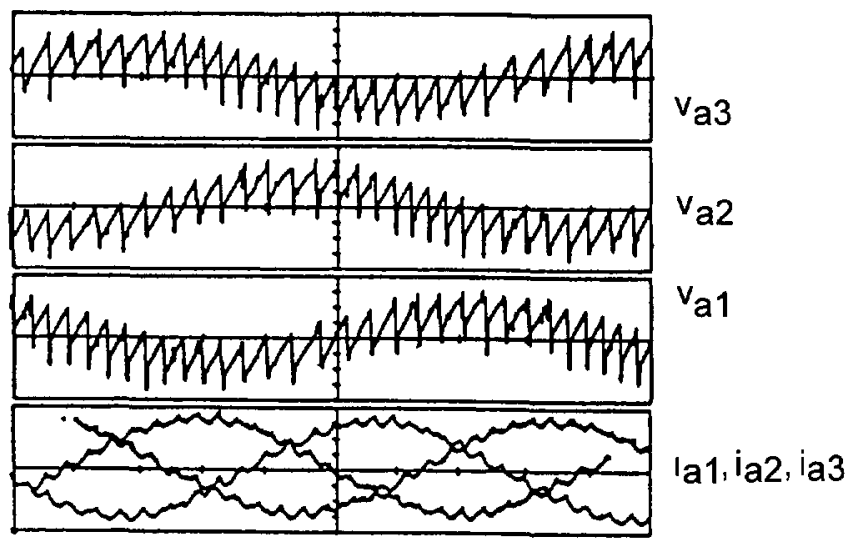

Fig 10. - Tensions/courants de sortie $\mathcal{R}=0,75 ; \cos \varphi_{0}=0,65 ; f_{0}=12,5 \mathrm{~Hz} ; 200 \mathrm{~V} /$ div , $10 \mathrm{~A} / \mathrm{div}, 10 \mathrm{~ms} / \mathrm{d} \mathrm{vv}$

[Output voltages/currents waveforms. $\mathcal{R}=075 ; \cos \varphi_{0}=0.65 ; f_{0}=125 \mathrm{~Hz}, 200 \mathrm{~V} / \mathrm{duv}, 10 \mathrm{~A} / \mathrm{div}$; $10 \mathrm{~ms} /$ div.]

0

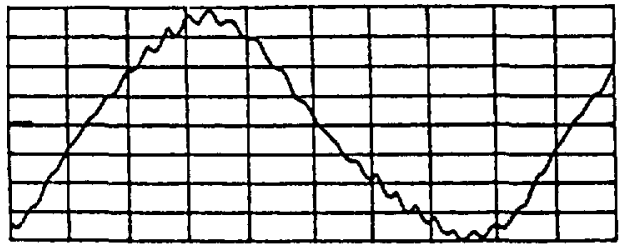

$\mathrm{Q}[\mathrm{t}]$ monophasée

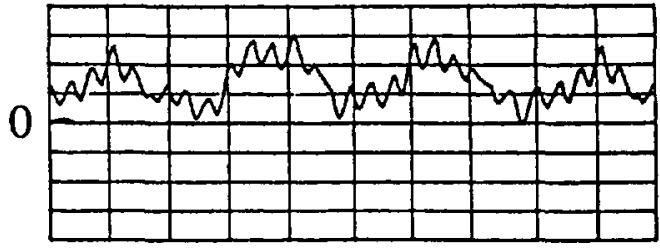

Q[t] triphașée

Fig 11. - Puissance réactive instantanée. $\mathcal{R}=0,75 ; \cos \varphi_{0}=0,65, f_{0}=12,5 \mathrm{~Hz} ; 4 \mathrm{kvar} / \mathrm{d} ı$, $10 \mathrm{~ms} / \mathrm{div}$.

[Instantaneous reactive power. $\mathcal{R}=0.75 ; \cos \varphi_{0}=0.65 ; f_{0}=12.5 \mathrm{~Hz} ; 4 \mathrm{kvar} / \mathrm{div}, 10 \mathrm{~ms} / \mathrm{dıv}$.]

\section{Conclusion}

Dans cet article, nous avons étudié et expérimenté des structures génériques de cycloconvertisseurs utilisant des interrupteurs à mécanismes de commutation duaux tels que le thyristor et le thyristor-diode-dual. Cette mixité des commutations permet de supprimer naturellement la puissance réactive moyenne au cœur du montage et assure une réversibilité totale vis-à-vis du courant de charge. Le montage en ponts complets bénéficie ainsi d'une augmentation très 


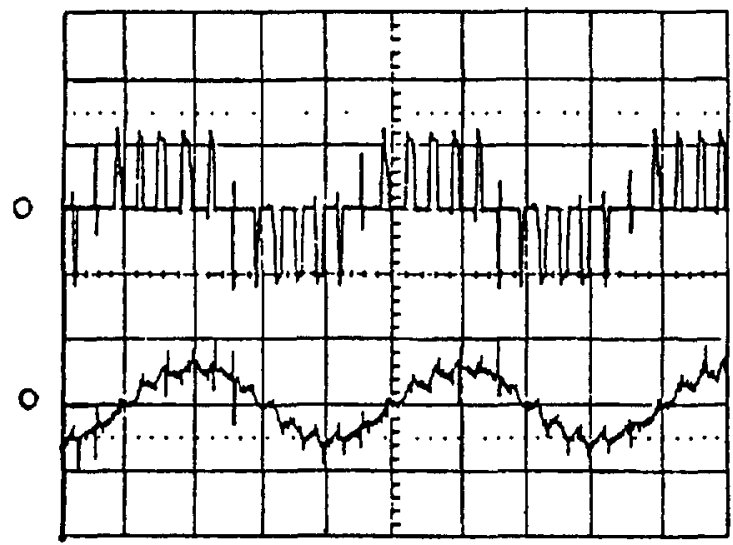

Tension de sortie

Fig. 12. - Tension/courant de sortie. $\mathcal{R}=0,47 ; \cos \varphi_{0}=0,65 ; f_{0}=12,5 \mathrm{~Hz} ; 500 \mathrm{~V} / \mathrm{div}, 20 \mathrm{~A} / \mathrm{div}$, $10 \mathrm{~ms} / \mathrm{div}$.

[Output voltages/currents waveforms. $\mathcal{R}=0.47 ; \cos \varphi_{0}=0.65 ; f_{0}=12.5 \mathrm{~Hz} ; 500 \mathrm{~V} / \mathrm{div}, 20 \mathrm{~A} / \mathrm{div}$, $10 \mathrm{~ms} /$ div.]

0

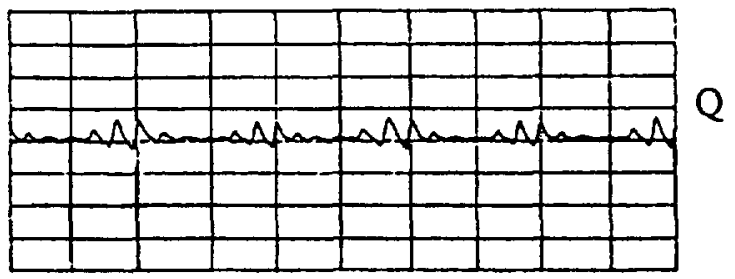

Fig. 13. - Puissance réactive instantanée $\mathcal{R}=0,47 ; \cos \varphi_{0}=0,65, f_{0}=12,5 \mathrm{~Hz} ; 1 \mathrm{kvar} / \mathrm{div}$; $10 \mathrm{~ms} / \mathrm{div}$.

[Instantaneous reactive power. $\mathcal{R}=0.47 ; \cos \varphi_{0}=065 ; f_{0}=12.5 \mathrm{~Hz} ; 1 \mathrm{kvar} / \mathrm{div} ; 10 \mathrm{~ms} / \mathrm{div}$ ]

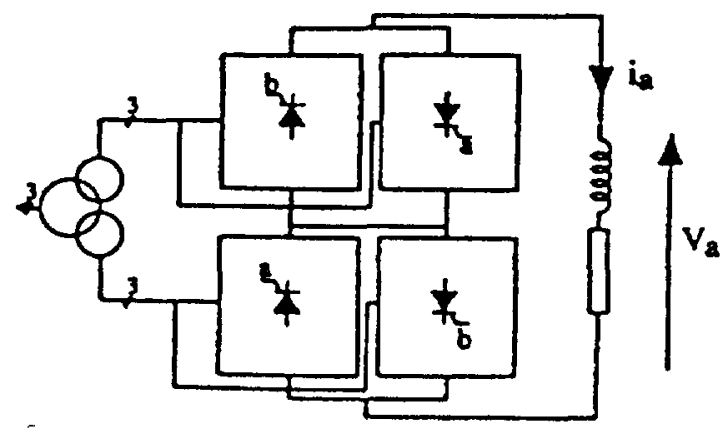

Fig. 14. - Mise en série de cycloconvertisseurs en ponts complets avec permutation des ponts.

[Full bridges series arrangement with permutation bridges] 


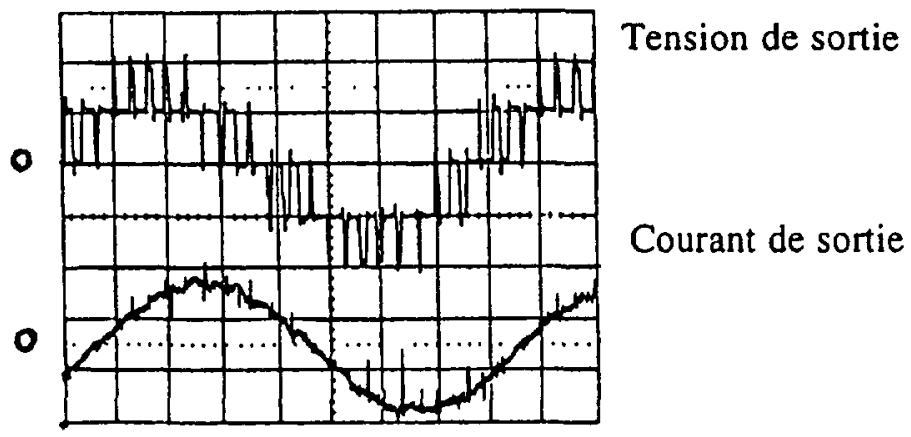

Fig. 15. - Tension/courant de sortie. $\mathcal{R}=0,70, \cos \varphi_{0}=0,70, f_{0}=12,5 \mathrm{~Hz} ; 500 \mathrm{~V} / \mathrm{div} ; 10 \mathrm{~A} / \mathrm{div}$, $10 \mathrm{~ms} / \mathrm{div}$.

[Output voltages/currents waveforms. $\mathcal{R}=0.70, \cos \varphi_{0}=0.70, f_{0}=12.5 \mathrm{~Hz} ; 500 \mathrm{~V} / \mathrm{div} ; 10 \mathrm{~A} / \mathrm{div}$; $10 \mathrm{~ms} / \mathrm{div}$ ]

importante du facteur de puissance mais produit des ondulations de puissance réactive à une fréquence triple de la fréquence de modulation. Cette imperfection peut toutefois être supprimée au moyen d'une association de montages mais requiert alors un nombre important de semiconducteurs.

\section{Remerciements}

Ces travaux ont fait l'objet d'un contrat de recherche DGA-DRET N89/223

\section{Bibliographie}

[1] Pelly B.R., Thyristor Phase-Controlled Converters and Cycloconverters (Wiley Interscience, 1971).

[2] Gyugyi L. and Pelly B.R., Static Power Frequency Changers - Theory - Performance and Application (Wiley Interscience, 1976).

[3] Gyugyi L., Input Power Factor of AC to AC Power Converters, IEEE Trans. on Industry Applications IA-16 (1980) 524-534.

[4] Chéron Y., La commutation douce dans la conversion statique de l'énergie électrique (Édition Technique \& Documentation, Lavoisier, 1989).

[5] Ladoux Ph., Chéron Y. and Foch Y., GTO and Duality - For a Better Matching of PhaseControlled Converters and Network, EPE J. 1 (1991) 113-124.

[6] Richardeau F., Commutateurs de courant et cycloconvertisseurs - Procédés de réduction et de contrôle interne de la puissance réactive, Thèse de doctorat de l'INP de Toulouse $\mathrm{N}^{\circ} 1164$ (1996). 